Reviews

\title{
Calculating Risks: The Spatial and Political Dimensions of Hazardous Waste Policy, by James T. Hamilton and W. Kip Viscusi, 1999, Cambridge, MA: MIT Press. 336 pp.
}

\section{Reviewed by Douglas L. Anderton, Social and Demographic Research Institute, University of Massachusetts-Amherst, Amherst MA.}

Hamilton and Viscusi's latest contribution to environmental policy studies is surely required reading for anyone with an interest in environmental policy, urban environmentalism or environmental equity. This volume presents a detailed account of the most thorough study yet of environmental risks and remediation policies for Superfund sites. It is a contribution both in terms of its substantive findings and in the author's exploration of methodology in a difficult terrain.

Despite the broad reach of the text's title, Hamilton and Viscusi's analyses are limited to the Superfund Program. That is an important qualification. While Superfund sites are a significant national concern, they present ex-post facto cases for many policy concerns that deal with initial siting and ongoing governance of environmentally hazardous enterprises. Among risk remediation policies, expenditures and public political interests, however, Superfund looms large. The authors offer a significant advance in Superfund assessment of great importance to pending legislative considerations.

Given the reliance of earlier studies on the EPA's own Hazard Ranking System, the relatively independent assessment of Superfund site risks (they still rely upon original record of decision data) by Hamilton and Viscusi is a substantial contribution. The authors' methodological efforts are as rewarding as their findings. Any hazardous risk assessment relies upon heroic assumptions, and the authors' assessments are not immune from such needs. Their assumptions involve, for example, homogenous population distributions within block or block-group areas; interpolative population growth estimates; risks independent of duration of residence; aggregative assumptions for exposure pathways in a population; and so forth. This is not to imply that their assumptions are especially problematic. To the contrary, the authors are most often conservative in their assumptions and provide a clear rationale for their decisions. The details of their sample construction and the assumptions involved in their risk assessment are outlined in two important appendices to the text that present a significant methodological contribution in their own right. Even if readers choose to disagree or dispute various assumptions, they will be rewarded and enriched by the authors' discussion of their methods. It is a rare text indeed where appendices are so well worth reading.

The authors' general conclusion regarding the extent of Superfund site risks, in the second and third chapters, is that risks are indeed substantial compared to other federally regulated risks but overstated in the EPA's calculations by (1) unlikely assumptions of future population immigration and growth in high risk areas, and (2) a compounding of sequentially conservative assumptions in risk calculation procedures. These two chapters (along with the appendices and Chapter Eight on market reactions to risk) are some of the strongest sections of the text. They are not, however, the most creative or intriguing chapters.

The next three chapters of the text venture into total risk assessment, cost-benefit computations and bounded EPA rationality. The extension of risks to population exposure in Chapter Four is a bold effort combining geographic information systems data management with strong assumptions to aggregate risks across population exposures. The authors' ambitious exercise clearly has a comparative advantage over EPA's calculations. And, in focusing on a 


\section{Reviews}

primary component of risks -cancer- the authors conclude that "once individual risk levels are combined with data on exposed populations, the magnitude of apparent cancer risks diminishes even further" (p. 108). Chapter Five carries this focus on cancer reduction into an assessment of the cost-effectiveness of EPA remediation policies. Across the sites studied, the authors find the median number of expected cancer cases over thirty years to be less than 0.1 with an estimated median cost per cancer averted, by past NPL policies, in the billions of dollars (using mean risk assumptions). Only about thirty percent of sites studied had a cost per cancer averted of less than $\$ 100$ million. Then, given these results, Chapter Six evaluates the rationality of EPA decisions from a risk reduction standpoint. The not surprising, but highly significant, conclusion reached by the authors is that "in hazardous waste cleanup decisions risk perception biases and risk politics matter...[and that]...greater scrutiny from residents pushes regulators away from decisions likely to maximize social welfare" (pp. 154-55).

Environmental equity is addressed in Chapter Seven using the risk measures and detailed remediation data constructed by the authors. The findings that methods of remediation selected vary by minority composition is one of substantial interest. It is unfortunate that the analysis does not definitively resolve the question as to whether these differences were in response to differences in community collective action, especially given the related finding that collective action was more intense at sites of lower risk. However, the authors are correct in suggesting that a policy of opening this decision process to greater scrutiny would potentially address these biases regardless of their source.

In a nicely crafted analysis, Chapter Eight brings in the consumer evaluation of risks through modeling housing prices. There are some limits to the modeling that might arise from endogeniety of area development trends or trajectories and Superfund developments. However, through analyzing price data before and after the release of EPA Remedial Investigation data, the authors conclude that consumers do learn from the data released and are able to incorporate this information into their own cost decisions.

All of these earlier results are brought together in Chapter Nine, which provides an overview of the authors' recommendations for revision of EPA site evaluations and the implementation of a risk-based assessment of the sort developed in the text. One clear contention is simply that EPA assessments have not had proper regard for population-based measures and cost assessments. The authors offer a simulation of remediation decision making under three policy scenarios including current policy, risk-based assessment only, and risk-based assessment with no assumption of future local population growth. The clear result of these simulations is that tremendous cost reductions could be made in Superfund remediation efforts through the use of a risk-based assessment with no, or only negligible, increases in risk. The primary effect of the assessment would simply be to focus high-cost remediations at sites with significant risk.

One substantial limitation to the analyses and policy recommendations is both intentional and consistent in this text - the reliance upon cancer cases as a metric of risk. The authors argue that the correlation between cancer risks and other morbidity risks is sufficient to merit cancer as an indicator variable (although it is treated more as an absolute measure than index) and that cancer risk is the most readily quantified of such indicators. There are, of course, many who might argue for the restrictiveness of this metric or for a more broad and inclusive risk metric. More generally there are those who, along with this reviewer, would question whether morbidity risk is the only social burden of concern in evaluating NPL sites. Although the authors amply demonstrate that risk information impacts personal property valuation, they do not establish the relative salience of risk. And, more to the point, they do not contend that risk is appropriate as the sole basis for social valuation. The primacy of cancer prevention as a risk metric in their analysis brings a reality to their evaluation that is a major step forward in Superfund research. However, cancer morbidity is certainly not the only, or only tangible, element of environmental site "cost" assessment.

Another possible concern with the authors' analyses is the potential for selection bias in the sample of NPL sites (i.e., 267 nonfederal sites with a record of decision signed in 1991-92) and with the smaller sub-sample used for risk assessment. One issue is the representativeness of the 
Reviews

1991-92 decision sites for other NPL sites. Since some studies have reported trends over time in the promotion of CERCLIS sites to the NPL (e.g., by community composition), there may be some concern that the 1991-92 sites are disproportionately residual sites that differ from those of earlier discovery and prioritization. This sampling decision introduces what are essentially crosssectional limitations to the analysis. A second limitation is raised by the exclusion, rather than stratification, of federal sites. Given their unique history and governance, there may well be a clear need to distinguish federal sites in analyses. However, the authors' omission of these sites is less than satisfactory for assessing hypotheses such as the distribution of site burdens among segments of the population. Finally, the fact that data for risk assessment were available for only 56 percent of the 1991-92 sites studied also presents some concern for selection biases. What sorts of sites did not have adequate data, and why? Although the authors do not answer this question directly, they do offer their assurances that the sub-sample is representative of nonfederal NPL sites in distributions by region, past site use and nature of contamination.

In such an ambitious endeavor as this text there are, of course, many less consequential assumptions and methods that can be questioned. The authors reach beyond their central thesis into a variety of related areas, e.g., environmental equity, hedonic land-use valuation, and so forth. And, it is difficult to exhaust all possible concerns with any one of these ambitious analyses within the context of a single chapter.

In terms of the analyses presented, the section of the text addressing environmental equity is the least well crafted. In this section, concern for detail is confused with a lack of theoretical and policy focus in approaching equity. Hamilton and Viscusi suggest that "[r]easoning from a single measure of exposure, one might conclude there is no 'environmental equity' problem in the Superfund program. . . [a]lternative risk indicators suggest there may, however, be a disproportionate minority exposure." And, "[d]ifferent definitions of minority populations are useful, since patterns of exposure may vary across different regions for groups such as blacks, Asians, Hispanics, and Native Americans." These statements are not problematic as a caution for researchers to have due regard for plausible multiple measures of risk and a recognition that ethnic groups are themselves diverse with regional distinctions. However, as a stricture for future research this advice leads to a loss of theoretical focus, causality and policy culpability. Strong inferential hypotheses such as discriminatory siting, "white flight," political and educational empowerment, etc., have all proven useful in equity studies precisely because they suggest a clear causal mechanism that can be tested and, if substantiated, addressed in a consistent policy fashion. Is the potentially spurious and sporadic finding that Hispanics in specific parts of the Southwest are more likely to live near industrial employment centers with Superfund sites sufficient to suggest a clear causal mechanism and pattern of environmental inequity requiring policy remediation? Such findings should be distinguished from strong inferential hypotheses suggesting, for example, that the disempowered at large are subject to environmental policy discrimination. If one examines multiple measures of exposure or risk and multiple population subgroups elaborated by region, ethnic group, income, education, etc., there is a virtual certainty of finding statistically, and substantively, significant findings in all directions due simply to the stochastic nature of the world within such a complex social lattice, the inadequacies of standard statistical methods for such data dredging, and the dwindling sample size and ahistoricism resulting from overelaboration.

Theoretical precision is more generally lacking in discussing environmental equity. For example, to test whether the higher concentration of minorities in urban areas is responsible for their greater concentration "near" (i.e., within four or ten miles) NPL sites, the authors compare the density distribution of block groups with NPL sites to those without NPL sites. This is a confounded and, at best, very indirect test of the hypothesis that involves minority residence only through assumed spurious correlation. Instead, this is a test of whether NPLs are put in the less densely populated areas on average. Density (especially within refined units of analysis) is a questionable indicator of urban location, especially if NPL sites are located within lower density 


\section{Reviews}

urban or higher density rural areas, if minorities are more often located within higher density rural areas, etc. In short, there is a confused connection between theoretical concerns and the evidence proffered. Similar confusion spills over into distinguishing earlier research addressing hypotheses of inequitable exposure from research addressing inequitable prioritization, and in distinguishing direct tests of strong inferential hypotheses from indirect suggestive descriptive findings. In short, the treatment of equity issues is not the authors' strongest contribution nor the most carefully crafted chapter of the work. These concerns, however, are largely limited to one chapter of the text which is arguably a diversion from the principal thrust of the analysis. The authors' central notion - that when evaluating risk population-based measures are appropriate - is acknowledged and distinguished as a specific hypothesis in prior equity research. None of these concerns detract from the central contribution of the text.

In the main, this text concerns the possible methods for a defensible assessment of Superfund site risks and the policy options for integrating such assessments into EPA procedures. There is little question that the authors have contributed in a profound way to this discussion. The authors' critique of EPA's existing policies is a major contribution. While many have questioned Superfund remediation costs, the authors have gone a step beyond others in a careful quantification substantiating these concerns. Their excursions into related issues, such as the role of risk information in housing valuation, raise interesting research questions and challenge existing conceptions of policy import. The simulation and assessment of policy options clearly establishes the salience of the policy options the authors offer. This text makes a clear contribution to Superfund policy research and also deserves serious attention by anyone concerned with the broader questions of society, economy and the environment.

\section{Property, Properties, and the Distribution of Wealth, A Review Essay by Stephen Gudeman, Department of Anthropology, University of Minnesota.}

\section{Property Relations: Renewing the Anthropological Tradition, edited by $\mathbf{C}$. M. Hann. 1998. Cambridge: Cambridge University Press. x, 277 pp.}

When landed wealth guaranteed position, and both were part of a predictable world, the conceit of property rights provided a taproot to social reality, because property ensured survival and status. With good reason, an earlier generation of anthropologists embraced the concept. Today, with globalization, the spread of post modernist ideas and the efflorescence of new wealth forms, the idea of property rights seems dated. The contributors to this volume, encouraged by C.M. Hann, are reviving this musty concept, by shifting the focus to property "relations" and bringing it to bear on new ethnography from around the world. Many of the results are interesting, but I found myself expanding their conceptual apparatus to include concepts about local models, the interaction of community and market in economy, distribution from a shared base or commons, and innovation.

What is the traditional wisdom? From Sir Henry Maine to Radcliffe-Brown and after, anthropologists emphasized that property refers to rights over things that people hold in relation to others. Property is not the object itself nor the relationship between person and object; rather, property specifies what a person can and cannot do with an article in relation to the rights held by others. This part of the traditional perspective is well explained in Chris Hann's Introduction and Jack Goody's contribution on dowry. For anthropologists, property is socially and legally 